\title{
Albertus Magnus, Super Iohannem (Ioh. 1, 1-18)
}

\section{Patrick Del Duca}

\section{OpenEdition}

\section{Journals}

Édition électronique

URL : https://journals.openedition.org/ccm/7920

DOI : $10.4000 / \mathrm{ccm} .7920$

ISSN : 2119-1026

\section{Éditeur}

Centre d'études supérieures de civilisation médiévale/Université de Poitiers

\section{Édition imprimée}

Date de publication : 1 septembre 2021

Pagination : 244-246

ISBN : 978-2-490783-10-6

ISSN : 0007-9731

\section{Référence électronique}

Patrick Del Duca, «Albertus Magnus, Super Iohannem (Ioh. 1, 1-18) », Cahiers de civilisation médiévale [En ligne], 255 | 2021, mis en ligne le 01 septembre 2021, consulté le 10 février 2022. URL : http:// journals.openedition.org/ccm/7920 ; DOI : https://doi.org/10.4000/ccm.7920

La revue Cahiers de civilisation médiévale est mise à disposition selon les termes de la Licence Creative Commons Attribution - Pas d'Utilisation Commerciale - Pas de Modification 4.0 International. 
Albertus Magnus, Super Iohannem (Ioh. 1, 1-18), Julie Casteigt (éd.), Louvain, Peeters Publishers (Eckhart, Texts and Studies, 10), 2019.

C'est un travail considérable qui a été accompli par Julie Casteigt avec cette édition critique d'une partie du commentaire de l'Évangile de Jean par Albert le Grand. Ce Dominicain, d'origine allemande et maitre de l'Université de Paris, est sans doute l'un des théologiens les plus marquants du XIII ${ }^{\mathrm{e}} \mathrm{s}$. En effet, après avoir enseigné à Paris, où il a eu comme élève le jeune Thomas d'Aquin, puis à Cologne, il a été évêque de Ratisbonne (1260-1262) avant d'abandonner sa charge pour se consacrer à nouveau à la prédication, à l'enseignement, et œuvrer à diverses entreprises de réconciliation. L'une de ses préoccupations essentielles aura été d'ouvrir le monde latin à la philosophie grecque, notamment à celle d'Aristote, 
qu'il a lui-même découverte à travers la tradition arabe de Cordoue.

Jusqu'à présent, le Super Iohannem n'était disponible que dans deux éditions anciennes, toutes deux disponibles en ligne (http://albertusmagnus.uwaterloo.ca/ Downloading.html [consulté le 05/10/21]) : celle de Pierre Jammy (Albertus Magnus, Opera omnia, vol. 11 : Super Iohannem, Lyon, Cl. Prost, 1651) et celle d'Auguste et Émile Borgnet (AlBertus Magnus, Opera omnia, vol. 24 : Enarrationes in Joannem, Paris, s. n., 1899). À la différence de ces deux volumes, la nouvelle édition scientifique de J. Casteigt ne reprend pas l'intégralité du texte d'Albert le Grand (qui occupe 807 pages dans l'édition de 1899). La portion éditée se limite au commentaire du Prologue de l'Évangile de Jean, c'est-à-dire des dix-huit premiers versets qui constituent la partie doctrinale du texte évangélique, précédant la partie historique qui commence au verset 19. Ce Prologue bien connu de Jean est consacré à l'incarnation du Verbe ainsi qu'au témoignage de l'évangéliste. Le commentaire d'Albert le Grand pose le problème de la connaissance : comment l'intellect humain, conjoint au continu et au temporel, peut-il percevoir ce qui ressortit du principe du Verbe éternel, de l'intellect paternel, " universellement agissant » (p. 15)? En ce sens, cette édition est essentielle pour comprendre un aspect important de la pensée de l'un des plus grands théologiens du Moyen Âge, d'un esprit hardi capable de concilier la philosophie d'Aristote et le christianisme, convaincu que tout ce qui relève de la raison est compatible avec la foi révélée dans les Écritures. En effet, Albert le Grand, s'appuyant sur l'autorité d'Augustin (Confessions VII, 9), considérait que le contenu du prologue de saint Jean, notamment l'idée du Verbe comme principe universel, se trouvait déjà dans les livres des philosophes, une différence essentielle demeurant toutefois celle de l'incarnation du Verbe, absente des textes anciens (cf. Verbe et Chair : les modalités de l'incarnation dans la réception philosophique de l'Évangile de Jean [Jn 1, 12-14], Julie CASTEIGt [éd.], Vienne/ Zurich, Lit Verlag [Théologie biblique, 1], 2018, p. 1). Par ailleurs, ce texte s'inscrit dans la tradition des traités doctrinaux commentant l'Évangile de Jean, à l'instar des commentaires ou homélies que Saint Augustin, Jean Chrysostome, Basile de Césarée ou encore Alcuin consacrent à ce texte.
Le travail réalisé par J. Casteigt se compose de deux parties et comprend tout d'abord une introduction longue de 244 pages. L'a. y pose la question de l'authenticité de l'œuvre et souligne que le nombre de catalogues médiévaux et de chroniques mentionnant le nom d'Albert le Grand comme auteur du Super Iohannem semblent bien attester la paternité de celui-ci. Elle aborde ensuite la question de la datation de l'œuvre et des circonstances qui ont accompagné sa naissance, revenant en détail sur l'hypothèse la plus souvent retenue : une première version de ce commentaire aurait « existé sous la forme d'une leçon donnée devant la curie pontificale [à Anagni] entre 1256 et 1257 » (p. XXXVIII). Après une analyse minutieuse des différentes positions historiographiques, elle conclut que cette hypothèse ne peut $a$ priori pas être exclue, ajoutant qu'une lecture précise de l'œuvre à travers les différents manuscrits ne permet pas de déterminer davantage les circonstances de la genèse du Super Iohannem. En ce qui concerne le titre de l'œuvre, l'a. indique que la plupart des manuscrits soit ne mentionnent aucun titre, soit se contentent de la mention de «Postilla ", terme désignant un commentaire exégétique de la Bible rédigé par des autorités patristiques. L'introduction contient également un aperçu détaillé des subdivisions logiques du texte, ce qui permet au lecteur de suivre plus facilement l'articulation qui sous-tend l'argumentation d'Albert le Grand. Il convient toutefois de noter que les titres et sous-titres donnés dans cet aperçu sont en latin. Un chapitre est consacré à la description des différents témoins contenant le commentaire du texte dans son intégralité ou seulement en partie : neuf manuscrits et quatre éditions imprimées contiennent le commentaire albertien complet. Le tout est complété par une carte permettant de mieux se représenter la diffusion géographique de l'œuvre, essentiellement limitée à l'espace germanophone. Le chap. $\mathrm{V}$, exhaustif, est consacré à la généalogie des témoins et à la reconstitution du texte : à partir de la comparaison de la totalité des leçons données par les neuf manuscrits conservés à ce jour, l'a. se livre à un examen des leçons communes, des divergences et des erreurs, afin de pouvoir reconstituer des liens généalogiques permettant de revenir au plus près du texte albertien d'origine, aujourd'hui perdu. Le stemma codicum ainsi obtenu lui permet de justifier le choix retenu pour l'établissement du texte édité. Le travail de collation effectué par J. Casteigt 
est impressionnant et permet d'arriver à certaines conclusions convaincantes. Ainsi, il s'avère que les manuscrits D E K G N sont sans doute éloignés de la leçon originelle et témoignent d'un état tardif de la réception de l'œuvre. En effet, l'a. démontre qu'il s'agit là d'œuvres corrigées et interprétées par des professeurs de théologie, des étudiants ou des dominicains œuvrant au milieu du $\mathrm{Xv}^{\mathrm{e}} \mathrm{s}$. dans le milieu rhénan. En cas de clivage entre différentes familles de manuscrits, l'éditrice privilégie donc, sauf erreur manifeste, les leçons restituant des strates plus anciennes ou les leçons les plus difficiles, reprenant les formules authentiques de l'a. avant qu'elles ne soient corrigées par la tradition rhénane plus tardive. L'analyse des éditions imprimées révèle que celles-ci témoignent de la réception du Super Iohannem dans le milieu rhénan du $\mathrm{Xv}^{\mathrm{e}} \mathrm{s}$. Cette analyse permet donc d'établir un archétype qui n'est corrigé que de manière très ponctuelle. Par ailleurs, se pose la question de la structure même du texte d'Albert le Grand. Ici encore, une analyse précise des indications marginales telles qu'elles apparaissent dans les manuscrits (notamment dans $\mathrm{Kl}, \mathrm{T}$ et $\mathrm{N}$ ) ainsi que la prise en compte des marques de chapitres permettent de dégager le plan du commentaire tel qu'il devait se présenter dans ses grandes lignes.

La deuxième partie de l'ouvrage contient le texte latin et, en regard sa traduction française. Le texte d'origine est accompagné de trois apparats : il est tout d'abord suivi des variantes propres aux différents manuscrits et textes imprimés, ainsi que des sources et des lieux bibliques. Le renvoi aux sources est précis et indique de la manière la plus exhaustive possible la littérature patristique dont s'est inspiré Albert le Grand, que celle-ci soit mentionnée explicitement par l'a. ou non. La traduction, tout en se voulant la plus proche possible du texte latin, n'en demeure pas moins d'un accès aisé et agréable pour un lecteur moderne.

L'édition bilingue est suivie de plusieurs indices : index des lieux bibliques, des auteurs, des passages cités dans l'appareil de notes, des thèmes et des mots contenus dans le commentaire. La deuxième partie contient également trois appendices comprenant le répertoire des leçons erronées, celui des variantes commentées ainsi qu'un appendice mentionnant les manuscrits qui contiennent des extraits ou des gloses du Super Iohannem. L'ouvrage se termine par une bibliographie générale et une table des matières.

Il convient donc de souligner la très grande qualité du travail fourni par J. Casteigt, tout juste pourra-t-on regretter l'utilisation à notre sens abusive de la numérotation romaine, la numérotation arabe étant réservée aux 319 pages comprenant l'édition bilingue et les index. Il nous semble que la numérotation arabe, si elle avait été appliquée à l'ensemble de l'ouvrage, n'aurait nui ni à sa lisibilité ni à sa cohérence. Un autre regret touche le choix de la portion traduite : certes, l'ampleur de l'œuvre d'Albert le Grand rend difficile une édition bilingue intégrale et le choix du prologue se justifie par la portée doctrinale de ce celui-ci. Néanmoins, peut-être aurait-il été bienvenu d'ajouter les deux prologues qui précèdent le commentaire, à savoir un Prologue à la louange de l'Évangéliste (Prologus in quo laudatur Evangelista) et une Explication du prologue de saint Jérôme à l'Évangile de Jean (Prologi Sancti Hieronymi in Evangelium secundum Joannem Explanatio), textes qui n'occupent que dix pages dans l'édition de 1899. En effet, le premier prologue compare les propriétés de l'aigle à celle de l'apôtre Jean s'élevant dans la contemplation du Verbe divin, tandis que le second préambule évoque les vertus de l'a. de l'Évangile et comporte des éléments sur le rapport qu'entretient, selon Albert le Grand, le texte biblique avec la philosophie des Anciens. Ces deux prologues, abordant la question de l'accès à la connaissance du principe premier, ainsi que le commentaire des versets 1 à 18 constituent les trois textes liminaires au commentaire évangélique lui-même. Ces trois parties forment un ensemble cohérent sur le fond, même si la forme distingue les deux premiers prologues $\mathrm{du}$ début du commentaire, et auraient donc pu, à notre sens, être regroupés dans cette nouvelle édition. Ces deux remarques demeurent cependant mineures et n'ôtent rien à la qualité remarquable de ce travail d'édition.

Patrick Del Duca LRL - Université Clermont Auvergne 